\title{
Advances in Immunotherapy for Metastatic Merkel Cell Carcinoma: A Clinician's Guide
}

Kelly G. Paulson, MD, PhD, , a,b,c and Shailender Bhatia, MDa,b,c

\begin{abstract}
Merkel cell carcinoma (MCC) is a neuroendocrine skin cancer. The clinical impact of MCC has been increasing due to steadily rising incidence rates. Since 2001, more than 24,000 cases of MCC have been reported to the US National Program of Cancer Registries database, and in 2018, more than 2,500 incident cases are expected. MCC is highly aggressive, and one-third of patients will either present with or develop metastatic disease. Outcomes in patients with metastatic MCC have historically been poor; median time to progression with cytotoxic chemotherapy is only 3 months. MCC has long been appreciated to be immunogenic, with reports of spontaneous regression and responsiveness to immunotherapy. However, the mechanisms of this immunogenicity have only been understood over the past decade, with approximately $80 \%$ of cases in the United States associated with the Merkel cell polyomavirus (MCPyV) and expression of viral antigens (virus-positive [VP] MCC), and the remaining $20 \%$ of cases caused by UV radiation-induced damage leading to a high mutational burden and expression of neoantigens (virus-negative [VN] MCC). These insights have led to multiple successful trials of immunotherapies for MCC. PD-1 axis checkpoint inhibitors are now regarded as the preferred frontline systemic therapy in eligible patients (including both VP- and VN-MCC), with impressive frequency, durability, and depth of objective responses, which compare favorably to those of most solid tumors. This article reviews the safety and efficacy data from the key clinical trials of immune checkpoint inhibitors for metastatic MCC, and discusses several issues relevant to the clinical use of these agents. Finally, emerging immunotherapies for MCC, including cellular therapies and adjuvant systemic therapies, are reviewed.
\end{abstract}

J Nat/ Compr Canc Netw 2018;16(6):782-790 doi: 10.6004ljnccn.2018.7049

\section{Merkel Cell Carcinoma Presentation and Incidence}

Merkel cell carcinoma (MCC) typically presents as a rapidly enlarging red nodule on sun-exposed skin. ${ }^{1}$ Risk factors include UV radiation exposure, age $>65$ years, and infection with the Merkel cell polyomavirus (MCPyV). Chronic systemic immune suppression (from HIV, hematologic malignancies, or use of immunosuppressive medications) is associated with increased MCC risk and significantly poorer outcomes ${ }^{1-3}$; however, most MCCs occur in individuals without underlying immunodeficiency. The reported incidence of MCC has been

aDivision of Medical Oncology, Department of Medicine, University of Washington; 'bred Hutchinson Cancer Research Center; and 'Seattle Cancer Care Alliance, Seattle, Washington.

Submitted February 4, 2018; accepted for publication May 15, 2018.

Dr. Paulson co-developed the described MCPyV serology assay, but receives no compensation related to this. She is also a coinventor on a patent related to a transgenic TCR in development for MCC. Dr. Bhatia reports research funding to his institution (University of Washington) from Merck, BMS, EMD-Serono, Oncosec, ImmuneDesign, and NantKwest, and has increasing rapidly, with approximately 25,000 cases recorded in the United States since 2000, including $>2,200$ incident cases reported in 2014 to the National Program of Cancer Registries/SEER combined registries, which captures $>98 \%$ of the US population (Figure $1 \mathrm{~A}) .{ }^{4}$ Incidence is expected to continue to briskly increase due to aging of the baby boomer generation, with $>3,000$ US cases estimated in 2025 . $^{5}$

The characteristic microscopic appearance for MCC is tightly packed, small, round, blue cells with high mitotic activity; immunohistochemistry is classically positive for cytokeratin 20 (CK20) in a dot-like pattern and

received honoraria for participation in advisory boards for EMD-Serono and Genentech.

This work was fully supported by the $\mathrm{NCl}$ of the NIH under award numbers T32CA009515 and P30CA015704. The content is solely the responsibility of the authors and does not necessarily reflect the official views of the NIH.

Correspondence: Shailender Bhatia, MD, Division of Medical Oncology, Department of Medicine, Seattle Cancer Care Alliance, 825 Eastlake Avenue E, Mailstop \#CE2-102, Seattle, WA 98109. Email: sbhatia@uw.edu 
A

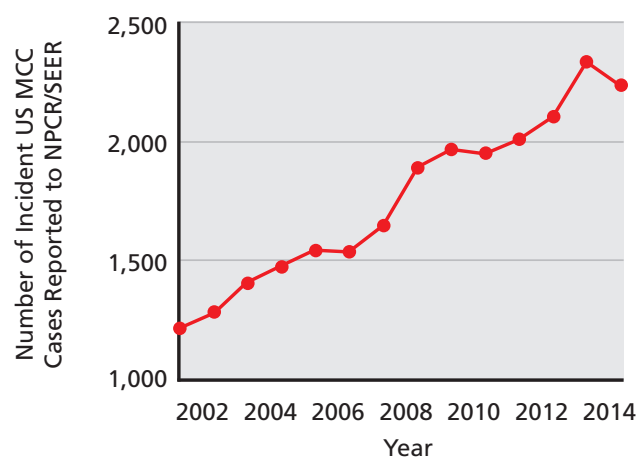

B

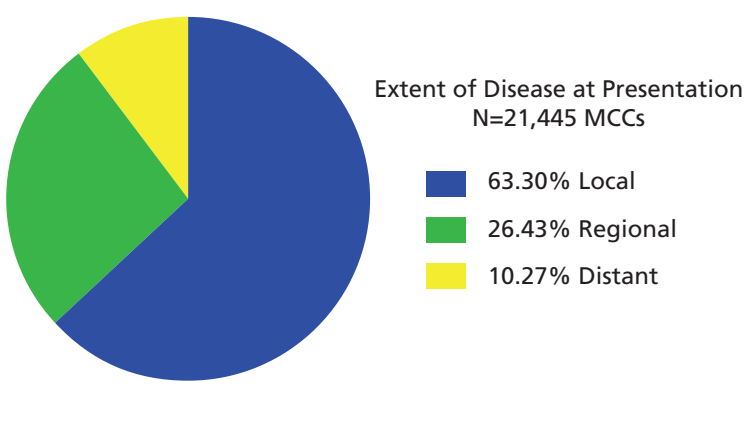

Figure 1. Incidence of Merkel cell carcinoma (MCC) in the United States. (A) Number of incident cases reported to National Program of Cancer Registries (NPCR)/SEER. Between 2001 and 2014, a total of 24,658 cases were reported. Annual number of incident cases increased by $>85 \%$, from 1,217 reported in 2001 to 2,246 reported in 2014. (B) Extent of disease at presentation. Information was available for 21,445 cases.

Data from 2001-2014 Database: National Program of Cancer Registries and Surveillance, Epidemiology, and End Results SEER*Stat Database: NPCR and SEER Incidence - USCS 2001-2014 Public Use Research Database, United States Department of Health and Human Services, Centers for Disease Control and Prevention and National Cancer Institute. Released August 2017, based on the November 2016 submission. Available at: www.cdc.gov/ cancer/npcr/public-use. Accessed June 15, 2018.

neuroendocrine markers (synaptophysin, chromogranin, and/or neuron-specific enolase), and negative for CK7 and TTF-1. Consistent with its histology, MCC is aggressive, and approximately $37 \%$ of patients have nodal or distant spread at presentation (Figure 1B); sentinel lymph node evaluation is thus frequently useful to identify subclinical nodal metastases. ${ }^{4,6}$ Systemic radiologic staging is encouraged by the NCCN Clinical Practice Guidelines in Oncology (NCCN Guidelines) for MCC in patients at high risk of distant metastatic disease. ${ }^{7}$ A case series of 102 patients with MCC showed improved sensitivity of whole-body FDG-PET compared with CT alone, with an added impact on management of $37 \%$ of patients. ${ }^{8}$

\section{Historical Outcomes With Metastatic Disease}

Approximately $10 \%$ of patients present with stage IV MCC (Figure 1B), and 1 in 3 patients will develop distant metastatic disease. ${ }^{9}$ Metastatic sites are diverse, including lymph nodes, liver, lung, subcutaneous tissue, and nonregional skin sites, as well as unusual sites such as pancreas, heart, and parotid. Brain metastases occur in approximately $10 \%$ of patients with metastatic MCC. ${ }^{10}$

Survival for patients presenting with stage IV MCC has been historically dismal, with a median survival of approximately 1 year and nearly $100 \%$ chance of death by 5 years. ${ }^{9,11}$ Until 2017, cytotoxic chemotherapy (typically with platinum/etoposide) was standard first-line therapy for metastatic MCC. Despite a high objective response rate (ORR) of approximately $55 \%$, durability of disease control is poor (median progression-free survival [PFS] of $\approx 3$ months and median duration of response $\leq 8$ months). ${ }^{3,12}$ After development of resistance to first-line therapy, second-line and later chemotherapy regimens have been ineffective, with an ORR of $10 \%$ and median PFS of only 1.9 months. ${ }^{3,12,13}$

\section{Two Distinct Immunogenic Etiologies for MCC}

Approximately $70 \%$ to $80 \%$ of MCC cases in the United States are caused by MCPyV, a small DNA tumor virus similar to human papillomavirus. ${ }^{14} \mathrm{Al}$ though MCPyV is common, with approximately 50\% of US individuals infected during childhood, it must develop 2 mutations (integration and truncation) to be oncogenic. ${ }^{15-18}$ Within virus-positive (VP) MCCs, the viral oncoproteins ( $\mathrm{T}$ antigens) are constitutively expressed and growth promoting. ${ }^{19-22}$ These nonhuman proteins are required for ongoing MCC growth, ${ }^{19}$ and are immunogens for $\mathrm{T}$ and $\mathrm{B}$ cells. ${ }^{3,23}$ Individuals with brisk immune responses to $\mathrm{MCPyV}$ have better 
disease outcomes, providing strong rationale for the application of immunotherapy. ${ }^{24-26}$

The remaining approximately $20 \%$ of MCCs are MCPyV-negative (virus-negative [VN] MCC). Because the main driver in VN-MCC is mutation from chronic UV exposure, it is more common in parts of the world with increased UV light exposure (eg, Australia). ${ }^{27} \mathrm{VN}$-MCC tumors carry a poorer prognosis, ${ }^{28}$ and thus a disproportionate percentage of patients who develop metastatic MCC have VN disease. ${ }^{29}$ Although they lack virus, VN-MCCs remain immunogenic due to high mutational burden from UV-induced mutations ${ }^{30-33}$; VN-MCCs have more mutations than almost all cancers in The Cancer Genome Atlas. ${ }^{30}$ High mutational and neoantigenic burdens have been reported to be strong predictors of immunotherapy responsiveness across cancer types, ${ }^{34}$ thus supporting application of immunotherapy in both VN- and VP-MCC.

The immunogenicity of MCC ${ }^{1,35}$ and PD-L1 expression in the MCC tumor microenvironment $t^{36,37}$ have provided a strong rationale for using immunotherapy. Efforts to date have focused on a multitude of approaches aiming to render cancer cells more visible to the immune system, reinvigorate existing immune responses, or generate new anticancer immunity. Indeed, several early-phase trials, including intratumoral interleukin-12 (IL-12), ${ }^{38}$ intratumoral TLR4 agonist, ${ }^{39}$ and MCPyV-directed adoptive T-cell therapy, ${ }^{40,41}$ have all supported the potential efficacy of immune-based approaches in MCC. However, the most striking successes have been with the PD-1 axis immune checkpoint inhibitors (ICIs) (Table 1), which have been remarkably efficacious and have revolutionized systemic therapy for MCC.

\section{PD-1 Axis Checkpoint Inhibitors}

\section{Avelumab}

Avelumab is a human anti-PD-L1 monoclonal antibody that maintains a wild-type $\mathrm{IgG} 1 \mathrm{Fc}$ region that may, in addition to blocking PD-1/PD-L1 interactions, activate antibody-dependent cell-mediated cytotoxicity. In a pivotal phase II trial, 88 patients with chemotherapy-treated metastatic MCC were treated with avelumab, $10 \mathrm{mg} / \mathrm{kg}$ intravenously every 2 weeks. The ORR was $33 \%(11 \%$ had a complete response [CR; $n=10$ ] and $22 \%$ had a partial response [PR; $n=19]) .{ }^{42,43}$ Responses occurred quickly

\begin{tabular}{|c|c|c|c|c|}
\hline & Avelumab & Pembrolizumab & Nivolumab & Ipilimumab \\
\hline $\begin{array}{l}\text { Mechanism } \\
\text { of action }\end{array}$ & Anti-PD-L1 & Anti-PD-1 & Anti-PD-1 & Anti-CTLA-4 \\
\hline $\begin{array}{l}\text { Dose \& } \\
\text { schedule of } \\
\text { administration }\end{array}$ & $\begin{array}{l}10 \mathrm{mg} / \mathrm{kg} \mathrm{IV} \\
\mathrm{q} 2 \mathrm{wk}\end{array}$ & $\begin{array}{l}2 \mathrm{mg} / \mathrm{kg} \\
\mathrm{q} 3 \mathrm{wk}\end{array}$ & $\begin{array}{l}240 \mathrm{mg} \\
\mathrm{IV} \text { q2wk }\end{array}$ & $\begin{array}{l}3 \mathrm{mg} / \mathrm{kg} \text { IV } \\
\text { q3wk } \times 4 \text { doses }\end{array}$ \\
\hline $\begin{array}{l}\text { ORR in } \\
\text { chemotherapy- } \\
\text { naïve MCC }\end{array}$ & $\begin{array}{l}69 \% \\
(n=16)^{45}\end{array}$ & $\begin{array}{l}56 \% \\
(n=26)^{29}\end{array}$ & $\begin{array}{l}71 \% \\
(n=14)^{46}\end{array}$ & $\begin{array}{l}40 \%(n=5 ; \\
\text { case series) }\end{array}$ \\
\hline $\begin{array}{l}\text { ORR in } \\
\text { chemotherapy- } \\
\text { treated/second- } \\
\text { line MCC }\end{array}$ & $\begin{array}{l}33 \% \\
(n=88)^{42,43,47}\end{array}$ & N/A & $\begin{array}{l}63 \% \\
(n=8)\end{array}$ & \\
\hline $\begin{array}{l}\text { Median time } \\
\text { to response }\end{array}$ & $6.1 w^{42}$ & $12 w^{29}$ & $2 \mathrm{mo}^{46}$ & \\
\hline
\end{tabular}

Abbreviations: IV, intravenous; MCC, Merkel cell carcinoma; N/A, not available; ORR, objective response rate.

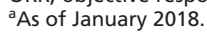

(median, 6.1 weeks) and irrespective of $\mathrm{MCPyV}$ or PD-L1 status of the MCC tumors (Figure 2). Importantly, responses were durable, with $74 \%$ lasting at least 1 year. As a result, median overall survival (OS) with second-line avelumab for metastatic MCC was 12.9 months, ${ }^{43}$ which was more than twice the historical median OS of 4 to 6 months with second-line chemotherapy. ${ }^{13,44}$ Avelumab was well tolerated. Although $70 \%$ of patients $(n=62)$ reported treatmentrelated adverse events (AEs), including most commonly fatigue (24\%) and infusion reactions (17\%), most were grade $1 / 2$; grade $3 \mathrm{AEs}$ were uncommon (5\%) and no grade 4 events were reported. Based on the impressive results from this study, avelumab received approval by the FDA, Swissmedic, and the European Medical Association in 2017 for treatment of metastatic MCC, regardless of prior chemotherapy administration. This trial has recently expanded to include treatment-naïve patients with metastatic MCC (ClinicalTrials.gov identifier: NCT02155647). Preliminary results of the first 39 patients enrolled in this first-line expansion cohort reported an ORR of $62 \%$ (CR, 14\%; PR, 48\%)..$^{45}$

\section{Pembrolizumab}

Pembrolizumab is a humanized IgG4 anti-PD-1 monoclonal antibody that is being investigated in a phase II trial for first-line systemic treatment of immunocompetent patients with unresectable stage IIIB or IV MCC (ClinicalTrials.gov identifier: NCT02267603). The initial report included 26 patients (25 evaluable patients), of whom $16 \%(n=4)$ had a CR and $40 \%(n=10)$ had a PR, resulting in an ORR of $56 \%$ 


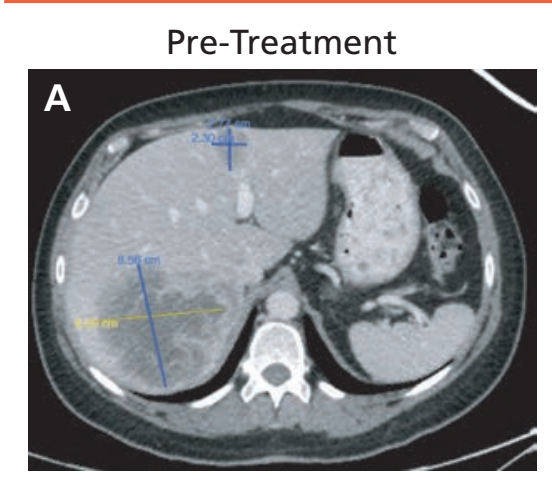

4.5 weeks after ICl start

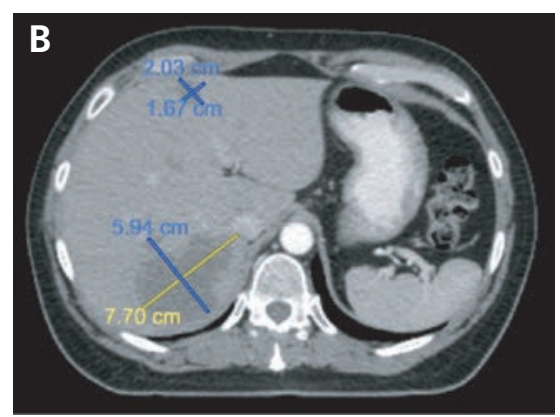

32 months after ICl start

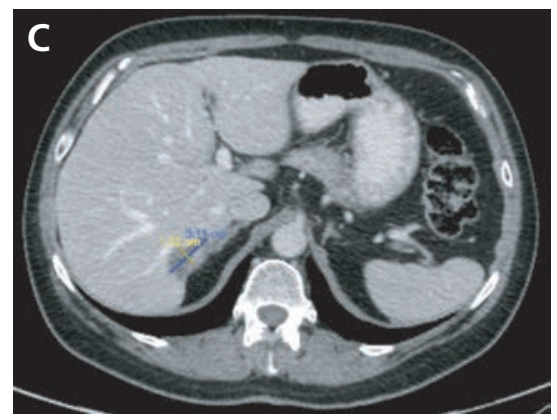

Figure 2. Rapid and durable response to avelumab in bulky liver metastases in a patient with MCPyV-negative MCC. Shown are cross-sectional imaging of 2 representative hepatic metastases from a 58-year-old woman with MCPyV-negative, chemotherapy-treated MCC (virus-negative) who was treated with avelumab for 27 months. (A) Pretreatment image of hepatic meastases. (B) An objective response was seen as early as 4.5 weeks after starting avelumab, and treatment was ultimately stopped for logistical reasons. (C) Repeat imaging at 32 months demonstrated ongoing response. Abbreviations: ICl, immune checkpoint inhibitor; MCC, Merkel cell carcinoma; MCPyV, Merkel cell polyomavirus.

$(14 / 25) \cdot{ }^{29}$ Although the response rate was similar to historical rates associated with first-line chemotherapy, pembrolizumab responses were remarkably more durable, with $86 \%$ ongoing at last follow-up (median follow-up $\approx 8$ months). Treatment was well tolerated: $77 \%$ of patients $(20 / 26)$ reported an AE of any grade, of which only $15 \%(n=4)$ were grade 3 or 4 . An expansion cohort of 24 patients has completed accrual and the results are eagerly awaited.

\section{Nivolumab}

Another ongoing study (ClinicalTrials.gov identifier: NCT02488759) is investigating the anti-PD-1 antibody nivolumab in patients with virus-associated cancers including MCC. Patients with metastatic $\mathrm{MCC}$ are eligible regardless of $\mathrm{MCPyV}$ status or prior chemotherapy. Preliminary results reported an impressive $68 \%$ ORR in 22 patients. ${ }^{46}$ The median time to response was 2 months, and $87 \%$ of responses were ongoing at last follow-up (median follow-up, 6 months). This trial has added a second cohort investigating nivolumab plus low-dose ipilimumab $(1 \mathrm{mg} / \mathrm{kg})$ in metastatic MCC.

\section{PD-1 Axis Blockade}

The striking and concordant results from the aforementioned trials of 3 different immunotherapies blocking the PD-1/PD-L1 interaction have offered powerful new options for managing advanced MCC. Responses to ICIs occur rapidly (Figure 2) and at a frequency similar to that expected with frontline chemotherapy, but are more durable, have been shown to be associated with improved quality of life, ${ }^{47}$ and are expected to lead to a meaningful improvement in OS, with perhaps even a chance at cure for an otherwise terminal disease. Importantly, response rates are higher in treatment-naïve patients and lower in those treated with prior chemotherapy, likely due to the well-characterized immunosuppressive effects of chemotherapy. ${ }^{48}$ Rates of grade $\geq 3$ treatmentrelated AEs have been low, and compare favorably with those of cytotoxic chemotherapy. Thus, PD-1/ PD-L1-based immunotherapy should be considered as the new standard of care for first-line treatment of patients with metastatic MCC. This consideration is reflected in the recent listing of avelumab, pembrolizumab, and nivolumab as the recommended options for disseminated MCC in the 2018 NCCN Guidelines, although a clinical trial should still be the preferred choice, whenever available and appropriate. ${ }^{7}$ Avelumab is currently the only FDA-approved therapy for patients with metastatic MCC.

For patients eligible for a PD-1 axis ICI, no clear data favor one drug over another; ORR looks comparable in the first-line setting. Avelumab has been tested most extensively so far and has also a demonstrated a quality-of-life benefit. ${ }^{47}$ However, both pembrolizumab and nivolumab are reasonable alternatives, with pembrolizumab administered every 3 weeks (vs every 2 weeks for nivolumab and avelumab) if a less frequent dosing schedule is preferred. Rates of toxicity also appear similar for the drugs, as does toxicity management. Management of immune-related AEs has been extensively detailed in the recent ESMO and Society for Immunotherapy of Cancer (SITC) guidelines. ${ }^{49,50}$ 
Contraindications to ICIs include ongoing immune suppression (likely to reduce efficacy), prior solid organ transplant, and/or prior autoimmune disease. Experience with ICIs in these settings is absent for MCC and limited for other malignancies. ICIs post-solid organ transplant have been reported to be associated with organ rejection and death. ${ }^{51}$ For autoimmune disease, a systematic review collated the effects of ICI therapy in 123 patients and found that $75 \%$ of patients with autoimmune disease developed flare of autoimmune disease and/or immune-related AEs. ${ }^{52}$ Most AEs were manageable with treatment discontinuation and steroids, but some were severe and fatal. Therefore, in these settings, the unique toxicity risks must be weighed against the risks of progressive MCC, particularly with the paucity of effective nonimmunotherapy options for such patients.

\section{Predictors of Response}

Nearly half of MCCs do not respond to PD-1 axis blockade. Thus, an urgent need exists to identify tumor or host characteristics that might predict nonresponse in order to prioritize clinical trials for these patients. Patients with both VP- and VN-MCCs (with markedly different tumor mutational burdens) appear to respond similarly to ICI therapy, reflecting the immunogenicity of both subsets, albeit through different mechanisms. ${ }^{29,42}$ Therefore, testing for MCPyV status (by immunohistochemistry or serology) or tumor mutational burden has limited utility in patient selection for PD-1 blockade. Similarly, ICI responses do not correlate with PD-L1 expression in the MCC tumor microenvironment, and thus PD-L1 expression testing by immunohistochemistry is not routinely supported. ${ }^{29,42}$ As for many other cancers, including melanoma, ${ }^{53}$ research is ongoing to identify new response biomarkers for ICIs in MCC because predictive biomarkers represent an unmet need.

\section{Metastatic MCC Resistant to PD-1 Axis Blockade}

In addition to identifying predictive biomarkers, mechanistic studies to understand both primary and acquired mechanisms of resistance are critical to uncover new rational therapies. A substantial need exists for effective therapies for MCC that does not respond to PD-1/PD-L1 blockade. Fortunately, several immunotherapy and nonimmunotherapy approaches are being pursued.

\section{Immunotherapy}

Early-phase trials and case reports have reported responses to a number of additional immunotherapies in MCC. Excitingly, several trials of novel immunotherapeutic approaches for MCC (both innate and adaptive) are underway.

Systemic Immunotherapy: Ipilimumab (antiCTLA4) has been used off-label in 5 patients with 2 successes. ${ }^{54}$ Prospective trials of ipilimumab (either alone or in combination with radiation and/or nivolumab) are ongoing (ClinicalTrials.gov identifiers: NCT03071406, NCT02488759) and results are eagerly anticipated. NCT02488759 includes a nivolumab plus anti-LAG3 arm, and a study of an IL-2 immunostimulatory antibody plus paclitaxel is ongoing in Europe (NCT02054884). Further, combination of anti-OX40 and nivolumab or ipilimumab has recently opened in the United States (NCT03241173). Given the subtle differences between the different drugs and in their targeted pathways, with anti-PD-1 agents targeting PD-1:PDL1 and PD-1:PD-L2 interactions compared with antiPD-L1 agents targeting PD-1:PD-L1 and B7.1:PD-L1 interactions, ${ }^{55}$ there may be a theoretical reason to consider switching drug class from an anti-PD-1 to an anti-PD-L1 agent or vice versa. However, in our experience, which is consistent with the limited data in melanoma and renal cell carcinoma, ${ }^{56}$ this strategy by itself is highly unlikely to be successful in overcoming MCC resistance to the PD-1/PD-L1 pathway blockade. Hence, we do not recommend trying the same outside of a clinical trial. However, there may be value in continuing the PD-1/PD-L1 pathway blockade, while adding other novel therapies on this backbone, to overcome immune evasion mechanisms.

Cellular Immunotherapy: The presence of viral antigens provides an ideal target for cellular immunotherapies. Endogenous T-cell therapy targeting $\mathrm{MCPyV}$ has been studied early-phase trials, with several responses reported, including durable $\mathrm{CRs}^{40,41}$; additional patients are accruing (ClinicalTrials.gov identifier: NCT02584829). A trial of NK cell immunotherapies in monotherapy or in combination with IL-15 has accrued (NCT02465957), with results 
pending, and a second multi-arm trial is anticipated to open in 2018 (NCT03167164).

Intralesional Immunotherapy: Multiple intralesional immunotherapies have been investigated in MCC. Case reports have indicated success with intralesional tumor necrosis factor, ${ }^{57,58}$ class I interferon, ${ }^{59-62}$ and talimogene laherparepvec (T-vec). ${ }^{63}$ In early-phase trials, intralesional TLR4-agonist and IL-12 delivery (via electroporation) have resulted in durable objective responses in patients with advanced MCC. ${ }^{38,39}$ Current active trials include T-vec alone or in combination with radiation (ClinicalTrials.gov identifier: NCT02819843) or nivolumab (NCT02978625), and TTI-621 (anti-CD47; NCT02890368). Additionally, a recently opened phase I/II trial of intralesional TLR7/8 agonist plus a modified IL-2 formulation is including patients with MCC (NCT03435640). A triple-combination study of tremelimumab (an anti-CTLA-4 antibody), durvalumab (an anti-PD-L1 antibody), and intratumoral TLR3 agonist poly-ICLC in advanced MCC is underway (NCT02643303).

Radiation as Immunotherapy: Single-fraction radiotherapy (SFRT) can be immunostimulatory, ${ }^{64}$ and such approaches have been effective for palliation/tumor control of one or a few lesions in MCC (objective in-field response rate of 94\%), ${ }^{65}$ although abscopal effects are not commonly seen in the absence of combination with systemic agents. ${ }^{66}$ SFRT is included as an immune adjuvant in many of the studies described earlier.

\section{Nonimmune Therapies}

Chemotherapy for MCC has typically included platinum doublet regimens similar to those for small cell lung cancer. Platinum and etoposide are most commonly used, with a response rate in first-line treatment of approximately $60 \%$, but a short PFS.,12 Preliminary results from a phase II trial of pazopanib reported an ORR of 19\%, but limited response durability. ${ }^{67} \mathrm{MCC}$ tumors express somatostatin receptors (SSTRs), responses to somatostatin analogs have been described, ${ }^{68}$ and trials delivering peptideconjugated payloads (ClinicalTrials.gov identifier: NCT02936323) and radionuclides onto SSTR-expressing neuroendocrine tumors are ongoing. Another trial (NCT02514824) is testing MLN0128, an mTOR inhibitor. ${ }^{69,70}$ Results from these nonimmune therapy trials are especially relevant to patients ineligible for immunotherapy.

\section{Resected MCC With High Risk of Recurrence}

Adjuvant radiation is supported in many cases of resected MCC, with strong evidence for improved locoregional control and a trend toward improved survival. ${ }^{6,71-74}$ However, adjuvant cytotoxic chemotherapy, despite being commonly used by clinicians, has not been associated with survival benefit. ${ }^{73,75,76}$ Given unclear benefit, considerable toxicity in an elderly population, and the potential negative effect on responsiveness to subsequent use of ICIs, ${ }^{42,45,47}$ adjuvant chemotherapy should not be used routinely. Fortunately, clinical trials are underway to test the safety and efficacy of ICIs as systemic adjuvant therapy in high-risk patients with MCC. A multicenter, randomized, placebo-controlled phase III trial investigating adjuvant avelumab in patients with clinically detected lymph node metastases (ADAM trial; ClinicalTrials. gov identifier: NCT03271372) was recently launched in the United States. Another trial, ADMEC-O (NCT02196961), is ongoing in Germany and is testing adjuvant ipilimumab (accrued) or nivolumab versus observation in patients with resected MCC.

\section{Surveillance}

After locoregional disease has been adequately treated, the NCCN Guidelines ${ }^{7}$ reflect a general clinical consensus and recommend a complete skin and lymph node examination every 3 to 6 months for the first 2 years, then every 6 to 12 months thereafter. Although radiologic surveillance is generally considered appropriate for patients at high risk for distant metastases, there is no strong consensus regarding which patients would be appropriate for radiologic surveillance, partly due to the lack of prospective data on recurrence-free survival in MCC. In practice, we typically do not perform routine surveillance imaging in nonimmunosuppressed patients with $<2-\mathrm{cm}$ primary MCC tumors and negative sentinel lymph node biopsy results because these groups have a $<25 \%$ risk of recurrence. ${ }^{11,77}$ Choice of imaging modality should reflect the sites at highest risk of recurrence. We typically use CT scans of the chest, abdomen, and pelvis in most patients, and sometimes CT scans of the head/neck and extremities, depend- 
ing on the location of the primary tumor and the risk of in-transit metastases.

Serology to T-oncoprotein is now clinically available (AMERK; https://www.merkelcell.org/serology). The NCCN Guidelines indicate that quantitation of $\mathrm{MCPyV}$ oncoprotein can help inform radiologic surveillance in patients with MCC, because seronegative patients may have a higher risk of recurrence, and an increasing titer in seropositive patients may be an early indicator of recurrence. ${ }^{26,78,79}$ If a patient is seropositive for viral oncoproteins at diagnosis, the antibody titer typically decreases after initial treatment and can increase with subsequent recurrence ${ }^{26,79,80}$; serial measurements may help inform the need for scans and reduce imaging-related radiation exposure.

\section{Special Considerations for the Geriatric Population}

A total of $83 \%$ of individuals diagnosed with MCC are aged $\geq 65$ years, and nearly a quarter of patients are $\geq 85$ years. ${ }^{7}$ ICI trials to date have largely reflected MCC demographics, with most enrolled patients aged $>65$ years, including very elderly individuals and even nonagenarians. ${ }^{29,42}$ Subgroup analyses have reported similar efficacy of ICIs in older populations. ${ }^{42}$ With quality of life being an important goal for the geriatric population, and given the relatively good tolerability of ICIs, these agents are the preferred choice for systemic therapy in this subset. For patients who are not medical candidates for surgery, radiation alone may be considered.$^{81}$ For those with advanced disease who wish to avoid the inconvenience and toxicity of a fractionated radiotherapy regimen, durable palliative control can often be achieved with SFRT with minimal toxicity ${ }^{65}$

\section{Conclusions}

MCC is a highly immunogenic, virus-associated skin cancer with a rapidly increasing incidence and clinical impact. Immunotherapy with PD-1 axis blockade leads to quick-onset, durable responses in metastatic MCC, and thus eligible patients should receive immunotherapy as first-line systemic therapy. Current research is centered around testing immunotherapy in the adjuvant setting for high-risk disease, investigating combinations to enhance ICI efficacy, and finding salvage therapies for patients with ICI-refractory disease. Learnings from immunotherapy successes and failures in MCC have high potential to guide immunotherapy efforts more broadly for all cancers and are worth aggressive pursuit.

\section{References}

1. Heath M, Jaimes N, Lemos B, et al. Clinical characteristics of Merkel cell carcinoma at diagnosis in 195 patients: the AEIOU features. J Am Acad Dermatol 2008;58:375-381.

2. Engels EA, Frisch M, Goedert JJ, et al. Merkel cell carcinoma and HIV infection. Lancet 2002;359:497-498.

3. Iyer JG, Afanasiev OK, McClurkan C, et al. Merkel cell polyomavirusspecific CD8(+) and CD4(+) T-cell responses identified in Merkel cell carcinomas and blood. Clin Cancer Res 2011;17:6671-6680.

4. 2001-2014 Database: National Program of Cancer Registries and Surveillance, Epidemiology, and End Results SEER*Stat Database: NPCR and SEER Incidence - USCS 2001-2014 Public Use Research Database, United States Department of Health and Human Services, Centers for Disease Control and Prevention and National Cancer Institute. Released August 2017, based on the November 2016 submission. Available at: www. cdc.gov/cancer/npcr/public-use. Accessed June 15, 2018.

5. Paulson KG, Park SY, Vandeven NA, et al. Merkel cell carcinoma: current United States incidence and projected increases based on changing demographics. J Am Acad Dermatol 2018;78:457-463.

6. Kim JA, Choi AH. Effect of radiation therapy on survival in patients with resected Merkel cell carcinoma: a propensity score Surveillance, Epidemiology, and End Results database analysis. JAMA Dermatol 2013;149:831-838.

7. Bichakjian CK, Olencki T, Aasi SZ, et al. NCCN Clinical Practice Guidelines in Oncology: Merkel Cell Carcinoma, Version 1.2018. Accessed May 24, 2018. To view the most recent version of these guidelines, visit NCCN.org.

8. Siva S, Byrne K, Seel M, et al. 18F-FDG PET provides high-impact and powerful prognostic stratification in the staging of Merkel cell carcinoma: a 15-year institutional experience. J Nucl Med 2013;54:1223-1229.

9. Fields RC, Busam KJ, Chou JF, et al. Five hundred patients with Merkel cell carcinoma evaluated at a single institution. Ann Surg 2011;254:465-475.

10. Kouzmina M, Koljonen V, Leikola J, et al. Frequency and locations of systemic metastases in Merkel cell carcinoma by imaging. Acta Radiol Open 2017;6:2058460117700449.

11. Asgari MM, Sokil MM, Warton EM, et al. Effect of host, tumor, diagnostic, and treatment variables on outcomes in a large cohort with Merkel cell carcinoma. JAMA Dermatol 2014;150:716-723.

12. Nghiem P, Kaufman HL, Bharmal M, et al. Systematic literature review of efficacy, safety and tolerability outcomes of chemotherapy regimens in patients with metastatic Merkel cell carcinoma. Future Oncol 2017;13:1263-1279.

13. Becker JC, Lorenz E, Ugurel S, et al. Evaluation of real-world treatment outcomes in patients with distant metastatic Merkel cell carcinoma following second-line chemotherapy in Europe. Oncotarget 2017;8:7973179741.

14. Feng $\mathrm{H}$, Shuda $\mathrm{M}$, Chang $\mathrm{Y}$, Moore PS. Clonal integration of a polyomavirus in human Merkel cell carcinoma. Science 2008;319:1096-1100.

15. Pastrana DV, Tolstov YL, Becker JC, et al. Quantitation of human seroresponsiveness to Merkel cell polyomavirus. PLoS Pathog 2009;5:e1000578.

16. Carter JJ, Paulson KG, Wipf GC, et al. Association of Merkel cell polyomavirus-specific antibodies with Merkel cell carcinoma. J Nat Cancer Inst 2009;101:1510-1522.

17. Chen T, Hedman L, Mattila PS, et al. Serological evidence of Merkel cell polyomavirus primary infections in childhood. J Clin Virol 2011;50:125129.

18. Shuda M, Feng H, Kwun HJ, et al. T antigen mutations are a human tumor-specific signature for Merkel cell polyomavirus. Proc Natl Acad Sci U S A 2008;105:16272-16277. 
19. Becker JC, Stang A, DeCaprio JA, et al. Merkel cell carcinoma. Nat Rev Dis Primers 2017;3:17077.

20. Kwun HJ, Shuda M, Feng $H$, et al. Merkel cell polyomavirus small $T$ antigen controls viral replication and oncoprotein expression by targeting the cellular ubiquitin ligase SCFFbw7. Cell Host Microbe 2013;14:125135 .

21. Shuda M, Chang Y, Moore PS. Merkel cell polyomavirus-positive Merkel cell carcinoma requires viral small T-antigen for cell proliferation. J Invest Dermatol 2014;134:1479-1481.

22. Shuda M, Kwun HJ, Feng H, et al. Human Merkel cell polyomavirus small $\mathrm{T}$ antigen is an oncoprotein targeting the 4E-BP1 translation regulator. J Clin Invest 2011;121:3623-3634.

23. Lyngaa R, Pedersen NW, Schrama D, et al. T-cell responses to oncogenic merkel cell polyomavirus proteins distinguish patients with Merkel cell carcinoma from healthy donors. Clin Cancer Res 2014;20:1768-1778.

24. Paulson KG, Iyer JG, Tegeder AR, et al. Transcriptome-wide studies of Merkel cell carcinoma and validation of intratumoral CD8+ lymphocyte invasion as an independent predictor of survival. J Clin Oncol 2011;29:1539-1546.

25. Miller NJ, Church CD, Dong L, et al. Tumor-infiltrating Merkel cell polyomavirus-specific $\mathrm{T}$ cells are diverse and associated with improved patient survival. Cancer Immunol Res 2017;5:137-147.

26. Paulson KG, Lewis CW, Redman MW, et al. Viral oncoprotein antibodies as a marker for recurrence of Merkel cell carcinoma: a prospective validation study. Cancer 2017;123:1464-1474

27. Garneski KM, Warcola AH, Feng Q, et al. Merkel cell polyomavirus is more frequently present in North American than Australian Merkel cell carcinoma tumors. J Invest Dermatol 2009;129:246-248.

28. Moshiri AS, Doumani R, Yelistratova L, et al. Polyomavirus-negative Merkel cell carcinoma: a more aggressive subtype based on analysis of 282 cases using multimodal tumor virus detection. J Invest Dermatol 2017;137:819-827.

29. Nghiem PT, Bhatia S, Lipson EJ, et al. PD-1 blockade with pembrolizumab in advanced Merkel-cell carcinoma. N Engl J Med 2016;374:2542-2552.

30. Goh G, Walradt T, Markarov V, et al. Mutational landscape of MCPyVpositive and $\mathrm{MCPyV}$-negative Merkel cell carcinomas with implications for immunotherapy. Oncotarget 2016;7:3403-3415.

31. Paulson $\mathrm{KG}$, Lemos $\mathrm{BD}$, Feng $\mathrm{B}$, et al. Array-CGH reveals recurrent genomic changes in Merkel cell carcinoma including amplification of L-Myc. J Invest Dermatol 2009;129:1547-1555.

32. Harms PW, Collie AM, Hovelson DH, et al. Next generation sequencing of cytokeratin 20-negative Merkel cell carcinoma reveals ultravioletsignature mutations and recurrent TP53 and RB1 inactivation. Mod Pathol 2016;29:240-248.

33. Harms PW, Vats $\mathrm{P}$, Verhaegen $\mathrm{ME}$, et al. The distinctive mutational spectra of polyomavirus-negative Merkel cell carcinoma. Cancer Res 2015;75:3720-3727.

34. Yarchoan M, Hopkins A, Jaffee EM. Tumor mutational burden and response rate to PD-1 inhibition. N Engl J Med 2017;377:2500-2501.

35. Paulson KG, Iyer JG, Blom A, et al. Systemic immune suppression predicts diminished Merkel cell carcinoma-specific survival independent of stage. J Invest Dermatol 2013;133:642-646.

36. Dowlatshahi M, Huang V, Gehad AE, et al. Tumor-specific T cells in human Merkel cell carcinomas: a possible role for Tregs and T-cell exhaustion in reducing T-cell responses. J Invest Dermatol 2013;133:1879-1889.

37. Afanasiev OK, Yelistratova L, Miller N, et al. Merkel polyomavirusspecific $\mathrm{T}$ cells fluctuate with merkel cell carcinoma burden and express therapeutically targetable PD-1 and Tim-3 exhaustion markers. Clin Cancer Res 2013;19:5351-5360.

38. Bhatia S, Iyer J, Ibrani D, et al. Intratumoral delivery of Interleukin-12 DNA via in vivo electroporation leads to regression of injected and noninjected tumors in Merkel cell carcinoma: final results of a phase 2 study [abstract]. Eur J Cancer 2015;51(Suppl 3):Abstract 504.

39. Bhatia S, Miller N, Lu HL, et al. Pilot trial of intratumoral (IT) G100, a toll-like receptor-4 (TLR4) agonist, in patients (pts) with Merkel cell carcinoma (MCC): final clinical results and immunologic effects on the tumor microenvironment (TME) [abstract]. J Clin Oncol 2016;34(Suppl 15):Abstract 3021.

40. Chapuis AG, Afanasiev OK, Iyer JG, et al. Regression of metastatic Merkel cell carcinoma following transfer of polyomavirus-specific $T$ cells and therapies capable of re-inducing HLA class-I. Cancer Immunol Res 2014;2:27-36.
41. Paulson KG, Perdicchio M, Kulikauskas R, et al. Augmentation of adoptive T-cell therapy for Merkel cell carcinoma with avelumab [abstract]. J Clin Oncol 2017;35(Suppl 15):Abstract 3044.

42. Kaufman HL, Russell J, Hamid O, et al. Avelumab in patients with chemotherapy-refractory metastatic Merkel cell carcinoma: a multicentre, single-group, open-label, phase 2 trial. Lancet Oncol 2016;17:1374-1385.

43. Kaufman H, Russell J, Hamid O, et al. Updated efficacy of avelumab in patients with previously treated metastatic Merkel cell carcinoma after $\geq 1$ year of follow-up: JAVELIN Merkel 200, a phase 2 clinical trial. J Immunother Cancer 2018;6:7.

44. Cowey CL, Mahnke L, Espirito J, et al. Real-world treatment outcomes in patients with metastatic Merkel cell carcinoma treated with chemotherapy in the USA. Future Oncol 2017;13:1699-1710.

45. D'Angelo SP, Russell J, Hassel JC, et al. First-line (1L) avelumab treatment in patients (pts) with metastatic Merkel cell carcinoma (mMCC): preliminary data from an ongoing study [abstract]. J Clin Oncol 2017;35(Suppl 15):Abstract 9530.

46. Topalian SL, Bhatia S, Hollebecque A, et al. Non-comparative, openlabel, multiple cohort, phase $1 / 2$ study to evaluate nivolumab (NIVO) in patients with virus-associated tumors (CheckMate 358): efficacy and safety in Merkel cell carcinoma (MCC) [abstract]. Cancer Res 2017;77(Suppl 13):Abstract CTO74

47. Kaufman HL, Hunger M, Hennessy $M$, et al. Nonprogression with avelumab treatment associated with gains in quality of life in metastatic Merkel cell carcinoma. Future Oncol 2018;14:255-266.

48. Harris J, Sengar D, Stewart T, Hyslop D. The effect of immunosuppressive chemotherapy on immune function in patients with malignant disease. Cancer 1976;37:1058-1069.

49. Haanen J, Carbonnel F, Robert C, et al. Management of toxicities from immunotherapy: ESMO Clinical Practice Guidelines for diagnosis, treatment and follow-up. Ann Oncol 2017;28:iv119-142.

50. Puzanov I, Diab A, Abdallah K, et al. Managing toxicities associated with immune checkpoint inhibitors: consensus recommendations from the Society for Immunotherapy of Cancer (SITC) Toxicity Management Working Group. J Immunother Cancer 2017;5:95.

51. Kittai AS, Oldham H, Cetnar J, Taylor M. Immune checkpoint inhibitors in organ transplant patients. J Immunother 2017;40:277-281.

52. Abdel-Wahab N, Shah M, Lopez-Olivo MA, Suarez-Almazor ME. Use of immune checkpoint inhibitors in the treatment of patients with cancer and preexisting autoimmune disease: a systematic review. Ann Intern Med 2018;168:121-130.

53. Jessurun CA, Vos JA, Limpens J, Luiten RM. Biomarkers for response of melanoma patients to immune checkpoint inhibitors: a systematic review. Front Oncol 2017;7:233

54. Winkler JK, Dimitrakopoulou-Strauss A, Sachpekidis C, et al. Ipilimumab has efficacy in metastatic Merkel cell carcinoma: a case series of five patients. J Eur Acad Dermatol Venereol 2017;31:e389-391.

55. Kim JM, Chen DS. Immune escape to PD-L1/PD-1 blockade: seven steps to success (or failure). Ann Oncol 2016;27:1492-1504.

56. Martini DJ, Lalani AA, Bosse D, et al. Response to single agent PD-1 inhibitor after progression on previous PD-1/PD-L1 inhibitors: a case series. J Immunother Cancer 2017;5:66.

57. Ito Y, Kawamura K, Miura T, et al. Merkel cell carcinoma. A successful treatment with tumor necrosis factor. Arch Dermatol 1989;125:10931095.

58. Hata Y, Matsuka K, Ito O, et al. Two cases of Merkel cell carcinoma cured by intratumor injection of natural human tumor necrosis factor. Plast Reconstr Surg 1997;99:547-553.

59. Paulson KG, Tegeder A, Willmes C, et al. Downregulation of MHC-I expression is prevalent but reversible in Merkel cell carcinoma. Cancer Immunol Res 2014;2:1071-1079.

60. Durand JM, Weiller C, Richard MA, et al. Treatment of Merkel cell tumor with interferon-alpha-2b. Br J Dermatol 1991;124:509.

61. Biver-Dalle C, Nguyen T, Touze A, et al. Use of interferon-alpha in two patients with Merkel cell carcinoma positive for Merkel cell polyomavirus. Acta Oncol 2011;50:479-480.

62. Wahl RU, Braunschweig T, Ghassemi A, Rubben A. Immunotherapy with imiquimod and interferon alfa for metastasized Merkel cell carcinoma. Curr Oncol 2016;23:e150-153.

63. Blackmon JT, Dhawan R, Viator TM, et al. Talimogene laherparepvec for regionally advanced Merkel cell carcinoma: a report of 2 cases. JAAD Case Rep 2017;3:185-189.

64. Kwilas AR, Donahue RN, Bernstein MB, Hodge JW. In the field: exploiting the untapped potential of immunogenic modulation by radiation in 
combination with immunotherapy for the treatment of cancer. Front Oncol 2012;2:104.

65. Iyer JG, Parvathaneni U, Gooley T, et al. Single-fraction radiation therapy in patients with metastatic Merkel cell carcinoma. Cancer Med 2015;4:1161-1170.

66. Cotter SE, Dunn GP, Collins KM, et al. Abscopal effect in a patient with metastatic Merkel cell carcinoma following radiation therapy: potential role of induced antitumor immunity. Arch Dermatol 2011;147:870-872.

67. Nathan PD, Gaunt P, Wheatley K, et al. UKMCC-01: a phase II study of pazopanib (PAZ) in metastatic Merkel cell carcinoma [abstract]. J Clin Oncol 2016;34(Suppl 15):Abstract 9542.

68. Fakiha M, Letertre P, Vuillez JP, Lebeau J. Remission of Merkel cell tumor after somatostatin analog treatment. J Cancer Res Ther 2010;6:382-384.

69. Slotkin EK, Patwardhan PP, Vasudeva SD, et al. MLN0128, an ATPcompetitive mTOR kinase inhibitor with potent in vitro and in vivo antitumor activity, as potential therapy for bone and soft-tissue sarcoma. Mol Cancer Ther 2015;14:395-406.

70. Snyder A, Makarov V, Merghoub T, et al. Genetic basis for clinical response to CTLA-4 blockade in melanoma. N Engl J Med 2014;371:2189-2199.

71. Jouary T, Leyral C, Dreno B, et al. Adjuvant prophylactic regional radiotherapy versus observation in stage I Merkel cell carcinoma: a multicentric prospective randomized study. Ann Oncol 2012;23:10741080.

72. Rush Z, Fields RC, Lee N, Brownell I. Radiation therapy in the management of Merkel cell carcinoma: current perspectives. Expert Rev Dermatol 2011;6:395-404.

73. Bhatia S, Storer BE, Iyer JG, et al. Adjuvant radiation therapy and chemotherapy in Merkel cell carcinoma: survival analyses of 6908 cases from the National Cancer Data Base. J Natl Cancer Inst 2016;108:djw042.
74. Mojica P, Smith D, Ellenhorn JD. Adjuvant radiation therapy is associated with improved survival in Merkel cell carcinoma of the skin. J Clin Oncol 2007;25:1043-1047.

75. Garneski KM, Nghiem P. Merkel cell carcinoma adjuvant therapy: current data support radiation but not chemotherapy. J Am Acad Dermatol 2007; 57:166-169

76. Poulsen M, Rischin D, Walpole E, et al. High-risk Merkel cell carcinoma of the skin treated with synchronous carboplatin/etoposide and radiation: a Trans-Tasman Radiation Oncology Group Study_-TROG 96:07. J Clin Oncol 2003;21:4371-4376.

77. Frohm ML, Griffith KA, Harms KL, et al. Recurrence and survival in patients with Merkel cell carcinoma undergoing surgery without adjuvant radiation therapy to the primary site. JAMA Dermatol 2016;152:10011007.

78. Paulson KG, Voillet V, Perdicchio M, et al. Acquired Cancer Resistance to Combination Immunotherapy from Transcriptional Loss of Class I HLA. Submitted.

79. Paulson KG, Carter JJ, Johnson LG, et al. Antibodies to merkel cell polyomavirus $\mathrm{T}$ antigen oncoproteins reflect tumor burden in merkel cell carcinoma patients. Cancer Res 2010;70:8388-8397.

80. Samimi M, Molet L, Fleury M, et al. Prognostic value of antibodies to Merkel cell polyomavirus $\mathrm{T}$ antigens and VP1 protein in patients with Merkel cell carcinoma. Br J Dermatol 2016;174:813-822.

81. Gunaratne DA, Howle JR, Veness MJ. Definitive radiotherapy for Merkel cell carcinoma confers clinically meaningful in-field locoregional control: a review and analysis of the literature. J Am Acad Dermatol 2017;77:142$148 \mathrm{e} 1$.

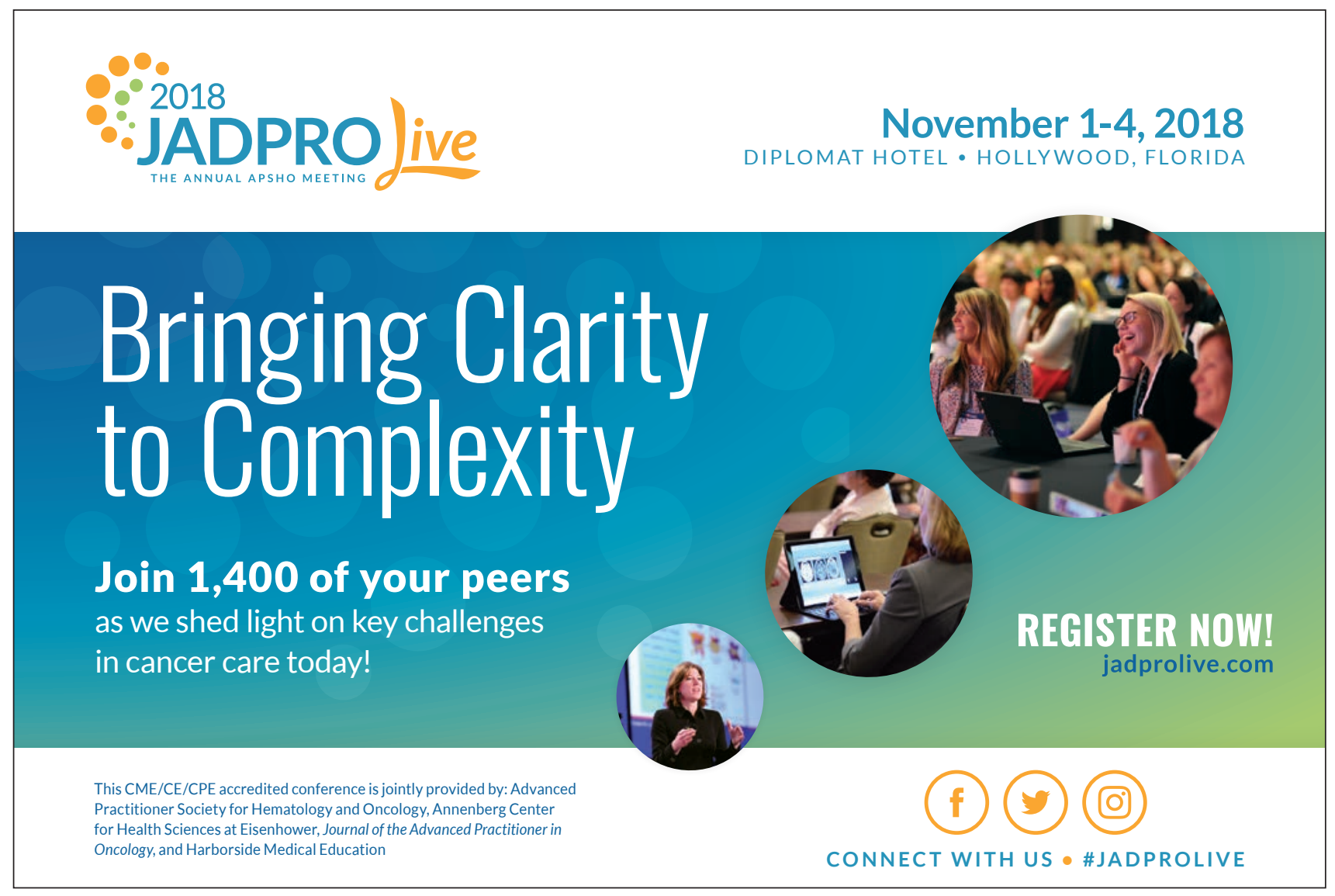

